\title{
RESERVA FLORESTAL DUCKE: DIVERSIDADE E COMPOSIÇÃO DA FLORA VASCULAR.'
}

\author{
José Eduardo Lahoz S. RIBEIRO², Bruce Walker NELSON³, Marlene Freitas \\ da SILVA ${ }^{4}$, Luisa Suely S. MARTINS ${ }^{5}$, Michael HOPKINS ${ }^{6}$
}

\begin{abstract}
RESUMO - A Reserva Florestal Ducke, localizada próxima a Manaus (AM), com uma área de $100 \mathrm{Km}^{2}$, é um dos locais com maior densidade de coletas depositadas no herbário do INPA. Sua flora vascular foi aqui caracterizada, através do levantamento de 7.107 exsicatas, das quais 4.946 estão determinadas ao nível de espécie. Embora muitas amostras estejam estéreis, indeterminadas e ainda não examinadas por especialistas, esta amostragem permitiu uma caracterização preliminar da flora da Reserva. Foi constatada a presença de 1.199 espécies (ou 1.453 , se incluídas as indeterminadas), distribuídas em 510 gêneros e 112 famílias. Os 10 gêneros mais diversificados -- com um total de 222 espécies -- são predominantemente arborescentes: Pouteria (38 espécies), Miconia (27), Protium (24), Licania (23), Inga (21), Ocotea (20), Swartzia (19), Eschweilera (19), Virola (16) Sloanea (15). As 10 espécies mais coletadas constituem apenas $8,2 \%$ do universo de amostras determinadas ao nível específico. Estas, também, são árvores: Micropholis guyanensis (A. DC.) Pierre, Chrysophyllum sanguinolentum (Pierre) Baehni, Trattinnickia glaziovii Swart, Goupia glabra Aubl., Scleronema micranthum Ducke, Aniba panurensis (Meissner) Mez, Laetia procera (Poeppig) Eichler, Caryocar villosum (Aublet) Pers.. Brosimum rubescens Taubert, Cecropia sciadophylla Mart. Apenas 123 espécies (10,3\% das espécies determinadas) são consideradas comuns, sendo aquelas com 10 ou mais coletas. A maioria das 1.199 espécies determinadas são muito raras: $68 \%$ representadas por três ou menos coletas e $43 \%$ representadas por apenas uma coleta. Portanto, muitas outras espécies "raras" serão acrescentadas após novas visitas ao local, ou através do estudo do material ainda indeterminado. É apresentada uma discussão e gráficos sobre as famílias e gêneros mais diversificados e mais coletados.
\end{abstract}

Palavras-chave: Levantamento florístico, Amazônia Central.

Ducke Forest Reserve: Diversity and composition of the vascular flora.

ABSTRACT - The Ducke Forest Reserve, with an area of $100 \mathrm{~km}^{2}$ and located close to Manaus, is one of the sites with highest density of botanical collections in the INPA herbarium. The reserve's vascular flora (excluding Pterophyta) is described based on a survey of approximately 7,107 specimens, of which 4,946 are determined to species. Though many specimens are sterile, undetermined, or not yet examined by specialists, this sample provides a preliminary floristic description of the reserve. The flora now stands at 1,199 species (or 1,453 if indeterminate are included), representing 510 genera and 112 families. The ten most speciose genera -- with a total of 222 species - are predominantly arborescent: Pouteria (38 species), Miconia (27), Protium (24), Licania (23), Inga (21), Ocotea (20), Swartzia (19), Eschweilera (19), Virola (16) e Sloanea (15). The ten most common (i.e., most collected) species comprise only $8.2 \%$ of the pool of determined specimens. These are also tree species: Micropholis guyanensis (A. DC.) Pierre, Chrysophyllum sanguinolentum

Projeto "Flora e Vegetação da Amazônia Central"- Convênio INPA/ODA.

2 Pesquisador Visitante, Convênio INPA/ODA. Instituto Nacional de Pesquisas da Amazônia/ CPBO - Caixa Postal 478, CEP 69.011-970, Manaus (AM).

3 Pesquisador. INPA/CPBO, Convênio INPA/ODA.

4 Professora Titular, Instituto de Tecnologia da Amazônia - UTAM-CEPEF, Av. Darcy Vargas, 1200.

5 Professora Adjunta, Instituto de Tecnologia da Amazônia - UTAN/CETEB.

6 Pesquisador Visitante, Convênio INPA/ODA, INPA/CPBO. 
(Pierre) Baehni, Trattinnickia glaziovii Swart, Goupia glabra Aubl., Scleronema micranthum Ducke, Aniba panurensis (Meissner) Mez, Laetia procera (Poeppig) Eichler, Caryocar villosum (Aublet) Pers., Brosimum rubescens Taubert,Cecropia sciadophylla Mart. Only 123 species ( $10.3 \%$ of the determined species) are considered common, being those with ten or more collections. The majority of the 1,199 determined species are very rare; $68 \%$ are represented by three or fewer collections and $43 \%$ are represented by a single collection. Therefore, many additional "rare" species will be added with additional collecting of the reserve or with the study of indeterminate material. Discussion and graphs comparing the most speciose and most commonly collected families and genera is included.

Key-words: Floristic Inventory, Central Amazonia.

\section{INTRODUÇÃO}

Apesar dos grandes avanços científicos nesse final de século, permanecemos, ainda, sem respostas à uma questão aparentemente muito simples, como: "Qual a composição florística de determinadas áreas geográficas?" Quando se pensa que a resposta a esta questão é básica para estudos de toda ordem, desde aqueles holísticos como os mais especializados, se tem idéia da importância da realização de inventários florísticos, pois é a partir desses trabalhos que passamos a ter uma base segura para a identificação da entidade biológica com a qual trabalhamos, além de informações adicionais como distribuição, fenologia, variação morfológica, preferência por habitat, formas de vida e aspectos econômicos. Numa outra escala, esse conhecimento nos oferece subsídios para estudos de fitossociologia, estratégias reprodutivas, biodiversidade, conservação e dinâmica de uma comunidade.

Uma Flora para toda a região amazônica seria o desejável. Entretanto, um trabalho desse porte, além de demorado, também exigiria grandes recursos financeiros e humanos. E, sob esse ponto de vista, a região de Manaus, e especificamente a Reserva Florestal Ducke, torna-se interessante sob vários aspectos: (1) devido ao grande número de coletas realizadas dentro e nas suas imediações (NELSON $e t$ al., 1990); (2) a proximidade com o INPA, o que permite o acúmulo de informações não só taxonômicas, além de possibilitar uma maior freqüência ao campo; e (3), principalmente pela sua localização, no centro da Amazônia, apresentando uma confluência das diferentes regiões fitogeográficas amazônicas (PRANCE, 1990). Além disso, CAMPBELL (1989) cita a região de Manaus como uma das prioritárias para a realização de inventários florísticos, devido, principalmente, à intensa ocupação humana na área.

A Flora da Reserva Ducke, idealizada devido à necessidade de um maior conhecimento de áreas representativas, através de coletas sistemáticas e confecção de monografias dos grupos amostrados, ao contrário de coletas esporádicas e envolvendo grandes extensões, é a primeira a ser iniciada para a Amazônia brasileira. As únicas obras disponíveis, atualmente, são a Flora Brasiliensis (MARTIUS, EICHLER \& URBAN, 1840-1906) que 
hoje está bastante desatualizada e que foi baseada em poucas coleções amazônicas e, mais recentemente, o trabalho de LEWIS \& OWEN (1989), que trata apenas das Leguminosas da Ilha de Maracá (RR). Este trabalho florístico na Reserva que, por si só, já acumula grande quantidade de informações, procura também oferecer subsídios básicos aos vários projetos em andamento, ou que venham a ser iniciados no local, além de servir de referência para toda a Amazônia Central.

\section{A Reserva Florestal Ducke}

Doada oficialmente ao INPA pelo governo do Estado do Amazonas, em 16 de fevereiro de 1963, a Reserva Ducke, compreendendo uma área total de $100 \mathrm{Km}^{2}$, dista apenas $26 \mathrm{Km}$ de Manaus (Fig.1). Trabalhos silviculturais na área tiveram início em 1960, e foram continuados até 1972, quando esses experimentos foram transferidos para outra Reserva na zona agrícola da SUFRAMA, localizada ao norte de Manaus, sendo então a Reserva Ducke declarada Reserva Biológica. Até 1991, menos de $5 \%$ da área da Reserva sofreu alterações visíveis em imagens de satélite, a maior parte na extremidade sul.

Estudos florísticos anteriores na Reserva Ducke foram apresentados por ALENCAR (1986) e por PRANCE (1990), PRANCE et al. (1976) também levantaram as árvores em um hectare de floresta de terra firme próxima da Reserva Ducke. O trabalho de ALENCAR (1986) engloba todas as árvores maiores de $10 \mathrm{~cm}$ DAP em 2,5 ha, sendo um total de 1.559 árvores distribuídas em 392 espécies, 156 gêneros e 53 famílias. PRANCE et al. (1976) contabilizaram 350 árvores de $15 \mathrm{~cm}$ DAP representando 179 espécies, 93 gêneros e 38 famílias. Quando incluída uma sub-parcela de $200 \mathrm{~m}^{2}$ para as árvores de $5 \mathrm{~cm}$ DAP, o total de espécies encontradas eleva-se para 235. MARTINS (1993) em um levantamento feito para a identificação de árvores e arbustos dióicos na Reserva, encontrou um percentual de $26,87 \%$ de famílias com espécies dióicas e cerca de $15,14 \%$ das espécies coletadas são de lenhosas que apresentam dioicismo.

PRANCE (1990) analisou uma listagem obtida a partir do banco de dados do Programa Flora (encerrado em 1982) onde estavam incluídas 825 espécies de plantas vasculares distribuídas em 91 famílias. Apesar de haver poucas coletas novas na Reserva Ducke após 1980, PAULA et al. (1989) concluíram que as informações obtidas do Programa Flora estavam ou desatualizadas, ou incompletas, conclusão esta reforçada pelos resultados aqui apresentados.

\section{Tipos de Vegetação na Reserva Florestal Ducke}

Quatro tipos de vegetação ocorrem na Reserva Ducke, cada uma associada com um elemento da paisagem. 


\section{Tipo de Vegetação/Espécies típicas \\ Mata de baixio:}

Oenocarpus bataua Mart.

Pouteria sp.

Socratea exorrhiza (Mart.) H.A.

Wendl.

Symphonia globulifera L.

Chrysophyllum sanguinolentum

(Pierre) Baehni

Rapatea paludosa Aublet

Hymenolobium sp.

Carapa guianensis Aublet

Allantoma lineata (Mart. ex Berg)

Miers

Mauritia flexuosa L. f.

Attalea spectabilis Mart.

Campinarana:

Pradosia schomburgkiana (A. de Candolle) Cronquist

Aldina heterophylla Benth.

Eperua glabriflora (Ducke) Cowan

Protium trifoliolatum Engl.

Pagamea sp.

Roucheria punctata Ducke

Duroia sp.

Coucpia racemosa Benth. ex Hook.f.

Humiria balsamifera (Aubl.) St. Hil.

Floresta de platô:

Dinizia excelsa Ducke

Caryocar villosum (Aublet) Pers.

Caryocar glabrum (Aublet) Pers.

Dipteryx odorata (Aublet) Willd.

\section{Paisagem/Ambiente/Estrutura}

Planície deposicional na margem de igarapé ou no fundo de páleo-lago; solo arenoso, encharcando com as chuvas, acúmulo de matéria orgânica; dossel 20-35 m de altura com muitas clareiras e poucas árvores emergentes.

Praias de páleo-lago retrabalhadas; coluvião; arenito meteorizado. Solo de areia quartzosa; alta penetração de luz, biomassa baixa; dossel 15-25 $\mathrm{m}$ de altura.

Platôs de pediplano não dissecado, solo argiloso de terra firme, bem drenado; biomassa alta; dossel 25-35 $\mathrm{m}$ altura com numerosas árvores emergentes $>45 \mathrm{~m}$ altura. 
Dipteryx odorata (Aublet) Willd.

Cariniana micrantha Ducke

Eschweilera sp.

Marmaroxylon racemosum (Ducke)

Killip

Duckeodendron cestroides Kuhlm.

Attalea attaleoides (Barb. Rodr.) W.

Boer

Floresta de vertente:

Dinizia excelsa Ducke

Oenocarpus hacaba Mart.

Astrocaryum sciophilum (Miq.) Pulle Eschweilera sp.

$\mathrm{Na}$ Reserva predomina a paisagem colinosa, dissecada da Formação Alter do Chão, com solos predominantemente argilosos. O pediplano erosivo original -- ou platô deposicional, segundo KLAMMER (1984) -- está preservado em poucos locais, como no interflúvio Acarál Bolívia próximo à margem ocidental da Reserva. Nestes platôs isolados ocorre floresta de maior biomassa e com maior número de emergentes, especialmente Dinizia excelsa. Suas copas largas excedem a 30 metros de diâmetro, criando uma textura visível, mesmo nas imagens Landsat, com pixel de $30 \times 30$ metros.

Segundo interpretação, em viagem de reconhecimento (W. SOMBROEK, com. pes. 1990), os solos de areia branca da Reserva Ducke seriam antigas praias e fundo de lagos. Estes solos estão localizados ao longo dos Igarapés Acará e Bolívia, tanto na
Paisagem colinosa, dissecada; solo argiloso ou areno-argiloso, bem drenado; poucas árvores emergentes acima do dossel de 25-35 $\mathrm{m}$ de altura.

terra firme comovas estreitas planícies encharcadas. A inundação destes dois igarapés ocorreu via represamento do rio Negro poucos metros acima de seu nível atual, possivelmente, na última fase interglacial. Estes braços do rio Negro seriam semelhantes ao igarapé Tarumã na atual fase interglacial (Holoceno) de represamento do rio Negro pelo mar. Outros braços fósseis de páleo-lagos ramificados são muito evidentes nas cabeçeiras do Rio Cuieiras, especialmente nos afluentes de sua margem esquerda.

Os solos arenosos de terra firme ao longo destes dois igarapés são cobertos por uma floresta típica de areia branca, a campinarana alta (ou caatinga amazônica arbórea, na classificaçāo de ANDERSON, 1981). Em alguns pontos, as árvores são muito finas, a penetração de luz é muito alta e o chão forrado de broméclias. Na margem esquerda do 
Igarapé da Bolívia existe uma pequena mancha com vegetação arbórea baixa, dominada por Pradosia schomburgkiana e Aldina heterophylla, esta última com seus galhos grossos cobertos de vegetação epifítica. É a vegetação físionomicamente mais próxima à "campina", existente na Reserva Ducke.

$\mathrm{Na}$ zona de inundação periódica destes dois igarapés e nos páleo-lagos preservados nas cabeceiras do Acará, ocorrem solos arenosos encharcados com acúmulo de matéria orgânica, devido às condições anaeróbicas. Neste ambiente ocorrem as matas de baixio.

A velocidade e a forma de dissecação nas vertentes argilosas são relacionadas com a densidade de pequenas falhas tectônicas, Existe uma linha com azimute de aproximadamente $349^{\circ}$ cortando a Reserva. A oeste desta linha, a paisagem está mais dissecada e as margens do páleo-lago são indistintas, A leste desta linha, a dissecação está menos ramificada e ainda são nítidas as margens do páleo-lago na alta bacia do Igarapé do Acará. Se o páleo-lago data da última fase interglacial, grande parte da dissecação se processou durante os últimos 100.000 anos, possivelmente sob clima mais seco, que teria estimulado uma erosão física acelerada.

Em imagens obtidas do sensor Thematic Mapper (TM) do satélite Landsat, é possível distinguir alguns dos elementos da paisagem e tipos de vegetação. Em composições falsa cor das bandas TM $3,4, \& 5$, as campinaranas e matas de baixio são uniformemente mais escuras, possivelmente devido à idade das folhas, ou então ao menor fechamento do dossel. As duas formações vegetais de terra firme e argilosa têm grande variação nos pixels, devido à irregularidades do dossel $\mathrm{e}$, também, devido à grande variação nas fases fenológicas. Em um pixel pode haver uma árvore sem folhas; no pixel ao lado uma árvore com folhas novas; e noutro pixel, árvore com folhas velhas cobertas de fungos escuros ou manchas de tecido seco e morto. Na vegetação do platô é possível detectar conjuntos de pixels correspondentes aos aglomerados de árvores emergentes, ou mesmo uma única árvore com copa de diâmetro $>50$ metros. Algumas manchas com emergentes também ocorrem esporadicamente sobre a vertente dissecada.

\section{MATERIAL E MÉTODOS}

Desde a implantação da Reserva Ducke, vários estudiosos têm amostrado sua flora, o que resultou em aproximadamente 7.107 números depositados no Herbário INPA. Entre 1988 e 1991, todas as exsicatas referentes aos espécimes coletados na Reserva Ducke foram levantadas, e as informações das etiquetas passadas para um fichário, acrescidos de dados contidos no livro de registros do herbário. Devido à necessidade de acesso rápido e praticidade no manuseio das informações contidas nas fichas, foi montado um banco de dados contendo todas as amostras 
coletadas na Reserva Ducke. Sendo um banco de dados preliminar, foram aceitas as determinações sem nova revisão taxonômica. Para algumas famílias, entretanto, as coletas da Reserva Ducke foram revisadas recentemente (por exemplo: Lecythidaceae, Chrysobalanaceae, Lauraceae, Burseraceae, Myristicaceae, Sapotaceae, Moraceae, Humiriaceae, Vochysiaceae, Annonaceae, Caryocaraceae, Arecaceae).

Todas as coleções de um determinado gênero, ainda não identificadas ao nível de espécie, entram no banco de dados com um único registro, como somente uma espécie, como por exemplo, Protium sp., com 6 coletas. Deste modo, o total de espécies obtido dentro das famílias e dos gêneros segue dois critérios: um total de espécies desconsidera as exsicatas indeterminadas ao nível específico, exceto quando todas as exsicatas daquele gênero forem indeterminadas. Neste caso, evidentemente, ocorre pelo menos uma espécie do gênero na Reserva; a outra soma obtida considera todas as exsicatas indeterminadas do gênero como mais uma (e apenas uma) espécie para aquele gênero. Para a ordenação das famílias e gêneros por número decrescente de espécies e de coletas utilizou-se da segunda soma obtida (número total das espécies, incluindo as indeterminadas). Isso não altera significantemente a mesma ordenação, quando comparada com a soma obtida excluindo os "taxa" indeterminados.

O sistema de classificação aqui aceito para as Angiospermas é o de
CRONQUIST (1981), o mesmo utilizado na organização do herbário INPA. Os nomes dos "taxa" e dos autores tiveram como referência, na ordem de prioridade para consulta: 1 ) monografias recentes; 2) material de herbário identificado por especialista e 3) o Index Kewensis. Foi montado um arquivo de referência para a grafia correta de todas as espécies determinadas, citando uma destas fontes.

O mapeamento da área foi realizado através de fotografias aéreas, estereoscópicas, na escala 1:50.000, obtidas em agosto de 1969 pela Empresa de Levantamentos Aerofotogramétricos Cruzeiro do Sul S.A. (Projeto O-235, fotos 633 à 636, 684 à 688 e 699 à 703). Tomou-se como base cartográfica a carta topográfica MI-579/ 1, de nome MANAUS-E, realizada pelo DSG em 1980, na escala de 1:50.000. Os nomes dos igarapés foram obtidos no INCRA - Manaus.

\section{RESULTADOS E DISCUSSÃO}

A flora vascular da Reserva Ducke encontra-se atualmente com 1.199 espécies coletadas e determinadas, distribuídas em 510 gêneros e 112 famílias. Incluindo-se as espécies indeterminadas como mais uma (e apenas uma) espécie para o gênero em questão, ocorre, atualmente, na Reserva. 1.453 espécies.

Analisando a listagem, observa-se que o número de 1.453 plantas vasculares excede em 628 "taxa", os resultados obtidos através de uma consulta ao banco de dados do 
Programa Flora (PRANCE, 1990), encerrado em 1982, que registrava para a Reserva Ducke apenas 825 espécies. PAULA et al: (1989) apontaram a desatualização deste banco, estimando, através de levantamento manual parcial, 1.354 espécies de plantas vasculares para a Reserva, já incorporadas no herbário do INPA.
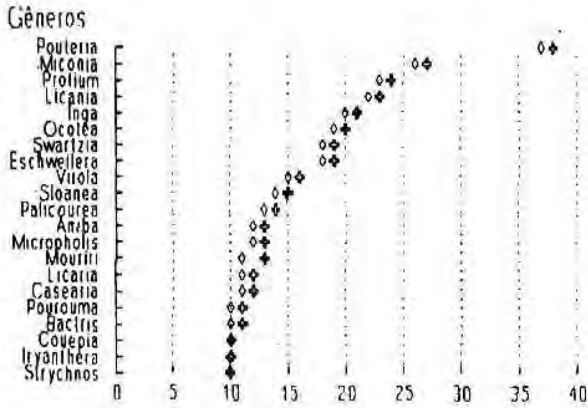

- EXCLLSPP.INDET \& INCL SPP.INIJET.

Figura 2. Gêneros mais diversificados na Reserva Florestal Ducke (EXCL.SSP.INDET. - excluindo espécies indeterminadas; INCL.SSP.INDET. - incluindo espécies indetermiadas).

Os 20 gêneros mais diversificados (Fig.2) correspondem aproximadamente àqueles revelados no banco de dados (PRANCE, 1990), com exceção de Licaria, Pourouma, Strychnos e Mouriri, que nesta versão mais atualizada, aparecem entre os mais ricos em espécies. Uma característica interessante dos gêneros encontrados na Reserva é que enquanto $21(4,1 \%)$ gêneros possuem 10 espécies ou mais, 305 (aproximadamente $59,8 \%$ ) gêneros são monoespecíficos.

O quadro mostrado por PRANCE (1990) para as famílias mais diversificadas continua o mesmo nesta nova listagem, com as 20 famílias mais ricas em espécies, diferindo apenas em suas posições (Fig. 3), com exceção de Flacourtiaceae, substituída

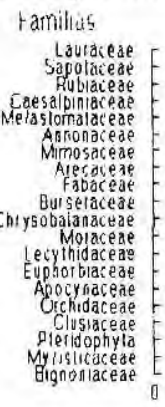

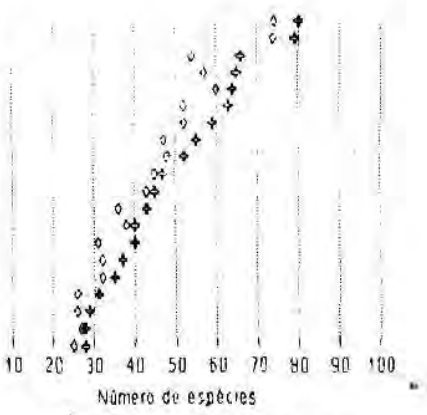

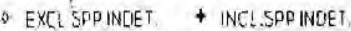

Figura 3. Famílias mais diversificadas na Reserva Florestal Ducke (EXCL.SSP.INDET. - excluindo espécies indeterminadas; INCL.SSP.INDET. - incluindo espécies indeterminadas).

por Clusiaceae nessa versão. A mesma tendência observada por PRANCE (1990) e GENTRY (1990) continua sendo notada nesta listagem mais atualizada, da maior ocorrência entre as mais diversificadas, de famílias formadas por "taxa" lenhosos. Com exceção de Orchidaceae, uma família principalmente epifítica; Rubiaceae e Melastomataceae principalmente arbustivas; Bignoniaceae e Clusiaceae com muitos representantes de hábito trepador e hemiepifítico, respectivamente, as demais famílias são predominantemente ou totalmente arbóreas.

As lianas são melhores representadas pelas famílias Bignoniaceae (27 spp.), Loganiaceae (12 spp.) e Menispermaceae (8 spp.). As famílias predominantemente herbáceas que se destacam pelo número de 
espécies são Cyperaceae (17 spp.), Poaceae (10 spp.) e Asteraceae (7 spp.). Entre as famílias predominantemente epifíticas, de'stacam-se Orchidaceae (35 spp.), Araceae (15 spp.) e Bromeliaceae (12 spp.).

Apesar do esforço dispendido até hoje, 516 espécies determinadas foram coletadas apenas uma vez e 819 espécies têm três ou menos coletas. Aquelas com 10 ou mais coletas somam apenas 123 "taxa" $(\mathrm{N}=4.946$

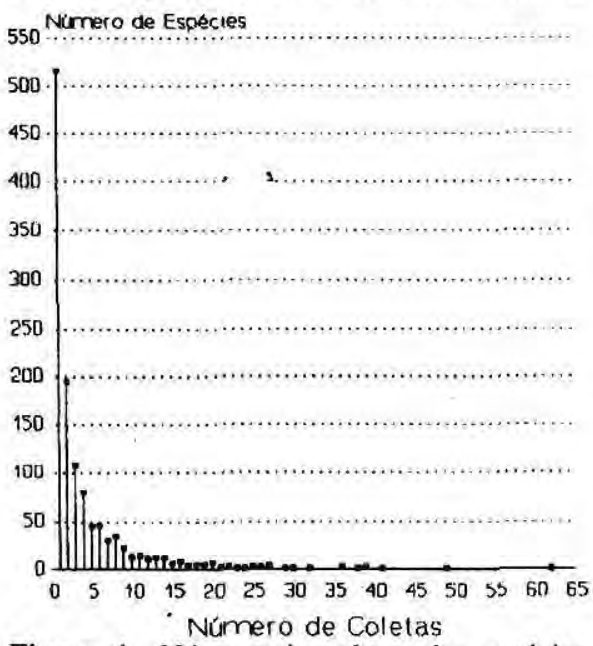

Figura 4. Número de coletas das espécies determinadas e depositadas no herbário INPA.

espécimes, $\mathrm{S}=1.199$ espécies; Fig. 4). Embora $31,36 \%$ das coletas estejam ainda indeterminadas ao nível de espécie, esses números demonstram que mais da metade das espécies são pouco freqüentes.

Já a nível de família, com exceção de Orchidaceae, Bignoniaceae e das Pterydophyta, as demais famílias mais coletadas são também as mais diversificadas (Fig.5). Espera-se que as famílias com maior número de "taxa" sejam também as mais

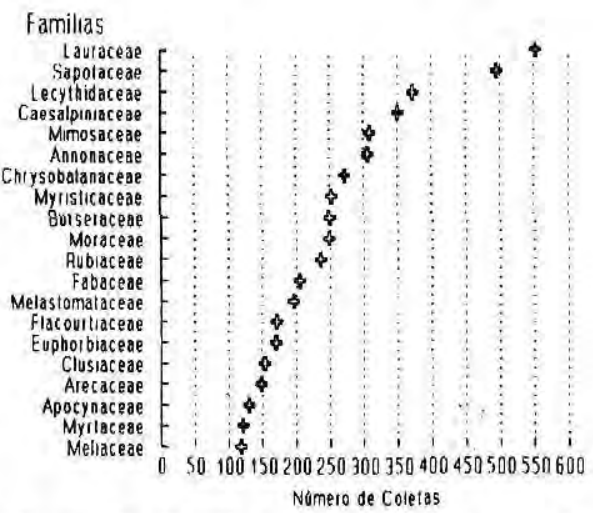

Figura 5. Famílias com maior número de espécimes depositados no herbário INPA.

coletadas. Mas, por outro lado, isso pode representar outro artefato de amostragem, onde as famílias mais coletadas, principalmente por especialistas, tendem a ter a sua diversidade melhor representada. Para os gêneros o quadro é semelhante, sendo que 14 dos 20 mais coletados

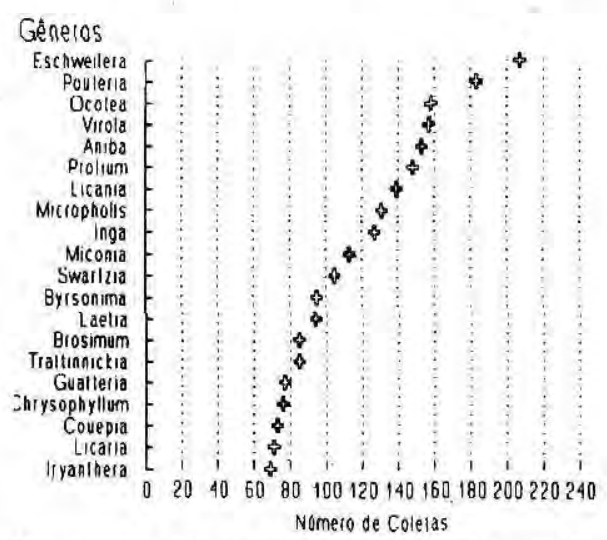

Figura 6. Gêneros com maior número de coletas depositadas no herbário INPA.

são também os mais diversificados (Fig.6).

PRANCE (1990) fez uma comparação entre a composição 
florística da Reserva Ducke e a das Reservas do Projeto DBFF, localizada a apenas $90 \mathrm{~km}$ ao norte de Manaus, chegando à conclusão de que há maior afinidade florística entre a flora das Guianas e a área do DBFF. Para a família Chrysobalanaceae, PRANCE (1990) contabilizou 26 espécies presentes na área do DBFF e ausentes na Reserva Ducke, mas, apenas 8 espécies presentes na Reserva Ducke e ausentes no área do DBFF. Possivelmente, estas diferenças apenas refletem efeitos de amostragem, considerando que o número de coletas de árvores na área do DBFF esteve

Tabela 1. Número de amostras identificadas a nível específico, genérico e de família, com o número e percentagem de amostras estéreis correspondentes.

\begin{tabular}{lccc}
\hline \multicolumn{1}{c}{ Amostras } & Total & Estéreis & $\%$ \\
\hline identificadas & 4946 & 2171 & 43,9 \\
Identificadas até gênero & 1511 & 1075 & 71,1 \\
Identificadas até familia & 650 & 581 & 89,4 \\
Total & $\mathbf{1 1 0 7}$ & 3823 & 53,8
\end{tabular}

aproximadamente 10 vezes maior que o número de coletas na Reserva Ducke em 1990. Haveria também maior número de coletas de arbustos e ervas na Reserva Ducke em relação ao projeto DBFF, explicando a falta de coleta de algumas espécies na área do DBFF. O grande número de espécies representadas por apenas uma coleta na Reserva Ducke sugere que, se a intensidade de amostragem fosse igual àquela do DBFF, haveria maior similaridade florística entre as duas áreas.

Ao lado dos números de coletas, que podem estar indicando artefatos de amostragem, alguns problemas são encontrados nos materiais analisados, como a falta de informações nas etiquetas, grande quantidade de material estéril (Tab.1) e coletas direcionadas para alguns tipos de hábitos, além do fato de algumas unidades vegetacionais permanecerem mal amostradas. Outra dificuldade encontrada é a adequação das diferentes terminologias referentes a ambientes e hábitos, utilizadas pelos diferentes coletores, impossibilitando muitas vezes a análise dessas informações.

O fato do número de "taxa" exceder ao número cadastrado pelo Programa Flora, além dos problemas acima apontados, demonstra a necessidade de realização de um trabalho sistemático tanto no campo quanto no herbário, conduzidos por uma equipe com esses objetivos, para uniformizar a informação já existente e acrescentar novas coletas e observações de campo.

Assim, esse quadro parcial será modificado com a obtenção de mais espécimes e observações de campo, e principalmente pelo estudo por especialistas do material já coletado.

\section{AGRADECIMENTOS}

Aos estagiários, bolsistas (CNPq) Antonio Ribeiro da Costa Neto, Maria do Socorro Ferreira dos Santos, Helena Cassia da Silva e, especialmente, a Jane Aparecida de Paula e Morgana Ferreira Aguiar, pelo paciente e trabalhoso levantamento manual das exsicatas depositadas no Herbário INPA, pela confecção do fichário e digitação de parte do banco de dados. 


\section{Bibliografia citada}

Alencar, J.C. - 1986. Análise de Associação e Estrutura de uma Comunidade de Floresta Tropical Úmida onde Ocorre Aniba rosaeodora Ducke (Lauraceae). Tese de doutoramento. INPA/FUA Manaus, Brasil, 332 pag.

Anderson, A.B. - 1981. White-sand vegetation of Brazilian Amazonia. Biotropica 13(3): 199-210.

Campbell, D,G, - 1989. The importance of floristic inventory in the tropics. In: CAMPBELL, D.G. \& HAMMOND, H.D. (eds.). Floristic Inventory of Tropical Countries. New York, New York Botanical Garden Publ. 545p.

Cronquist, A. - 1981. An Integrated System of Classification of Flowering Plants. Columbia University Press, . New York. $1.263 \mathrm{p}$.

Gentry, A.H. - 1990. Floristic similarities and differences between Southern Central America and Upper and Central Amazonia. In: A.H. GENTRY (ed.). Four Neotropical Rainforests. Yale University Press, New Haven. 627p.

Klammer, G. - 1984. The relief of the extraAndean Amazon basin. In: H. SIOLI (ed.). The Amazon: Limnology and landscape ecology of a mighty tropical river an its basin. Junk Publishers, Dordrecht. $763 \mathrm{p}$.

Lewis, G.P. \& Owen, P.E. - 1989. Legumes of the Ilha de Maracá. Royal Botanic Gardens - Kew. 95p.
Martins, L.S.S, - 1993. Dioicismo na Reserva Florestal Ducke, Manaus, AM. Dissertação de Mestrado. INPA/FUA, $111 \mathrm{p}$.

Martius, K. F. P. von; Eichler, A. W.; Urban, I. (eds.) - 1840 a 1906. Flora Brasiliensis. 15 volumes em 40 partes. 130 fascículos. Munique, Viena e Leipzig. 20.733 p.

Nelson, B. W.; Ferreira, C. A. C.; da Silva, M. F.; Kawasaki, M. L. - 1990. Endemism centers, refugia and botanical collection density in Brazilian Amazonia. Nature 845:714-716.

Paula, J.A.; Nelson, B.W. \& Silva, M.F. - 1989. Avaliação do banco de dados do Programa Flora para cadastramento das plantas vasculares da Reserva Florestal Ducke. Resumos do XL Congresso Nacional de Botânica. Cuiabá (MT), 22 a 28/01/1989. Vol. 2 .

Prance, G. T.- 1990. The floristic composition of the forests of central Amazonian Brazil. In: A. Gentry (ed.) Four Neotropical Forests. Yale University Press. New Haven. 627 p.

Prance, G.T.; Rodrigues, W.A. \& Silva, M.F. 1976. Inventário florestal de um hectare de mata de terra firme $\mathrm{km} 30$ da estrada Manaus - Itacoatiara. Acta Amazonica 6(1): 9-35. 